

1) Primary Health Care Centre of Kissamos, Chania, Crete, Greece

2) Clinic of Social and Family Medicine, Faculty of Medicine, University of Crete, Greece
DOI: $10.15386 / \mathrm{mpr}-1568$

Manuscript received: 08.01.2020

Received in revised form: 12.03 .2020

Accepted: 27.03.2020

Address for correspondence:

danyfantakis@yahoo.gr

This work is licensed under a Creative Commons Attribution-NonCommercialNoDerivatives 4.0 International License

\section{Diabetic neuropathy and restless legs syndrome: can a known chronic condition slow down our diagnostic way of thinking? A case report and a short literature overview}

\author{
Dimitrios Anyfantakis ${ }^{1}$, Fani Katsanikaki ${ }^{1}$, Emmanouil K. Symvoulakis ${ }^{2}$
}

\begin{abstract}
Restless legs syndrome is a common lifelong neurological disorder with a negative impact on the patients' sleep and quality of life. Despite its common occurrence in the general population, the condition usually remains undiagnosed. Patients with diabetes run at a higher risk to suffer from restless legs syndrome. Diabetic neuropathy, in particular, shares similar clinical characteristics with restless legs syndrome and reduces significantly the patients' quality of life.

Our aim was to report on an interesting case of restless legs syndrome in a patient with diabetes seeking medical advice for a ten-year long insomnia. Sleep deprivation was falsely attributed to diabetic neuropathy. A literature overview of the up-to-date knowledge was performed in order to summarize available information and provide primary care physicians with a comprehensive way of clinical thinking to differentiate peripheral neuropathy and restless legs syndrome manifestations.
\end{abstract}

Keywords: restless legs syndrome, diabetic neuropathy, diagnosis, primary care

\section{Introduction}

Restless legs syndrome (RLS) represents a chronic neurological movement and sleep disorder that burdens about $2-5 \%$ of the general population in Europe and the United States [1]. It is one of the most commonly detected reason of sleep deprivation with adverse effects on social and family life $[2,3]$.

Patients with diabetes are at higher risk to present RLS compared to the general population. Interestingly, RLS and diabetic neuropathy (DN), in particular, one of the most common complications of Diabetes Mellitus (DM), are two separate neurological conditions with some overlapping clinical manifestations [2] posing a clinical challenge for the physician.

We present a ten year diagnostically ignored case of RLS in a woman with DM admitted to a primary health care unit of rural Crete because of chronic sleep disorder, when this disturbance led to extreme distress.

\section{Case report}

A 65-year-old woman suffering from type II DM since the age of 35 years under treatment with insulin glargine 40UI and metformin $850 \mathrm{mg}$ twice daily, visited the Primary Health Care Centre of Kissamos, Chania, Crete, reporting insufficient sleep and common nocturnal awakenings for the last 10 years. Her past medical history was unremarkable except for hypertension and dyslipidemia.

At the same time, the patient reported a chronic burning sensation in the lower limbs that was relieved by movement and housework. Her insomnia was presumed to be due to hypoglycemia episodes or diabetic neuropathy, for which duloxetine was administered without improvement.

Neurological examination did not 
reveal any focal abnormal signs. Muscle strength measured on a 5 point scale was normal and $5 / 5$ at both sides (thigh and tibia bending / extensor control, limb elongation / tilting, landing / tipping). Muscle tone was also normal (passive bending control and joint extension as well as assessment of possible resistance). Achilles tendon reflex (plantar flexion, root S1) was symmetric and of equal intensity at both sides.

Laboratory investigations were normal except of HbAlc 8.4\% (>6.5\%); glucose $201 \mathrm{mg} / \mathrm{dl}(70-125 \mathrm{mg} / \mathrm{dl})$; hemoglobin $11.4 \mathrm{~g} / \mathrm{dl}$ (11.5-15.5); ferritin levels $9 \mathrm{ng} / \mathrm{dl}$ (13$151 \mathrm{ng} / \mathrm{dl}$ ). Thyroid function, vitamin B12, folic acid levels, renal, liver and immunological markers were all normal. Funduscopic examination was normal and without diabetic retinal lesions. Serum electrolytes [sodium $141 \mathrm{mmol} / \mathrm{L}$ (137147), potassium $4.6(3.5-5 \mathrm{mmol} / \mathrm{L})$, calcium $9 \mathrm{mg} / \mathrm{dl}(8.4-$ $10.2)$ and magnesium $1.8(1.3-2.1 \mathrm{mg} / \mathrm{dl})$ were also normal. Night time measurements of glucose were also requested in order to rule out episode of hypoglycemia.

Electromyography measures muscle response or electrical activity in response to a nerve's stimulation of the muscle. Electromyography of the patient's nerves was normal for latency, amplitude, conduction, velocity and F-waves.

Upon electrical stimulation of the tibial nerve, superficial fibular (peroneal) nerve and deep fibular (peroneal) nerve, no pathologic signal could be obtained after proximal and distal stimulation.

Fulfillment of five basic clinical criteria established the RLS diagnosis. Pramipexole $0.7 \mathrm{mg}$ was administered before bedtime along with gluconic iron supplement once daily.

Three weeks later, the patient's sleep habits were significantly improved, while discomfort in the lower extremities disappeared.

\section{Discussion}

RLS is a sensory motor neurological disorder, first reported from Sir Thomas Willis in the $17^{\text {th }}$ century [3,4]. Later, in the 1940s, Karl Ekbom, revisited this neurological dysfunction of the lower limbs occurring during rest [5]. Currently RLS is a leading cause of insomnia [2], with severe negative impact on personal, family and occupational life [3].

A female preponderance has been reported with an approximately double prevalence in women compared to men across all population and age groups $[1,2]$.

It is classified as primary RLS which is the most common with genetically driven determinants (early or late onset) and secondary RLS [6] associated with conditions such as pregnancy, renal failure, rheumatoid arthritis, iron deficiency and polyneuropathies [2]. Some medications such as calcium channel blockers, antidepressants, lithium, dopamine antagonists and caffeine, alcohol and tobacco can worse or trigger RLS [3].
Patients seek advice from doctors with complaints of insomnia, fatigue, distress or pain in the lower limbs [7]. They usually experience a night-time distressing urge to keep in motion their lower limbs [2]. This extreme need is frequently accompanied by an unpleasant sensation which can be described as "creeping", "crawling", "burning", "pulling" or "soda bubbling in the veins" [2]. The symptoms range from a simple distress to irritation and intense pain [2]. RLS symptoms usually get worse over time [2].

Diagnosis is based on the revised five essential diagnostic criteria by IRLSSG (International RLS Study Group) [8]: 1) A strong urge to move the legs, 2) Symptoms begin or get worse during intervals of rest or inactivity such as lying down or sitting, 3) Symptoms are partially or totally relieved by movement, such as walking or stretching, 4) Worsening of symptoms occurs during evening or at night (circadian variation), 5) Existence of the above 4 clinical features are not due to other medical or behavioral conditions.

The fifth criterion was introduced in 2012 in order to rule out conditions that mimic RLS (myalgia, venous stasis, leg edema, arthritis, leg cramps, positional discomfort, habitual foot tapping) [8].

Although the symptoms that are described occur frequently, and patients experience significant impairment and distress, RLS is still masked [3]. The diagnosis of RLS remains a clinical challenge [2]. This occurs because patients do not describe their distress accurately [2], neurological examination is unremarkable, symptoms may mimic other conditions and there are no pathognomonic laboratory RLS markers [2]. Additionally, medical awareness regarding RLS basic clinical features when these are described by the patient seems to be limited.

Despite the lack of existing evidence for a certain correlation between DM and RLS, diabetic population groups are at a higher risk of RLS compared to the general population ones [2,9].

DN and RLS are two distinct neurological disorders that often lead to clinical performance 'artefacts' [9]. This is because RLS and DM often coexist and share many clinical similarities. Symptoms of DN are non-specific and may include intermittent or continuous burning; itching, tingling, numbness in the lower limbs which may also appear in RLS cases. This may result to the misdiagnosis of RLS among diabetics [10], inadequate glycemic control, overuse and misuse of healthcare services [3]. Since pharmacological management of RLS and DN is different [10], symptoms are expected to endure.

Therefore, differential diagnosis is based on identifying the main differences between these two conditions $[2,10]$ which appear in Figure 1. Furthermore, $\mathrm{DN}$ is listed as a diagnosis of exclusion. Nerve conduction study examination could have a role in differentiating between DN and RLS [11]. 


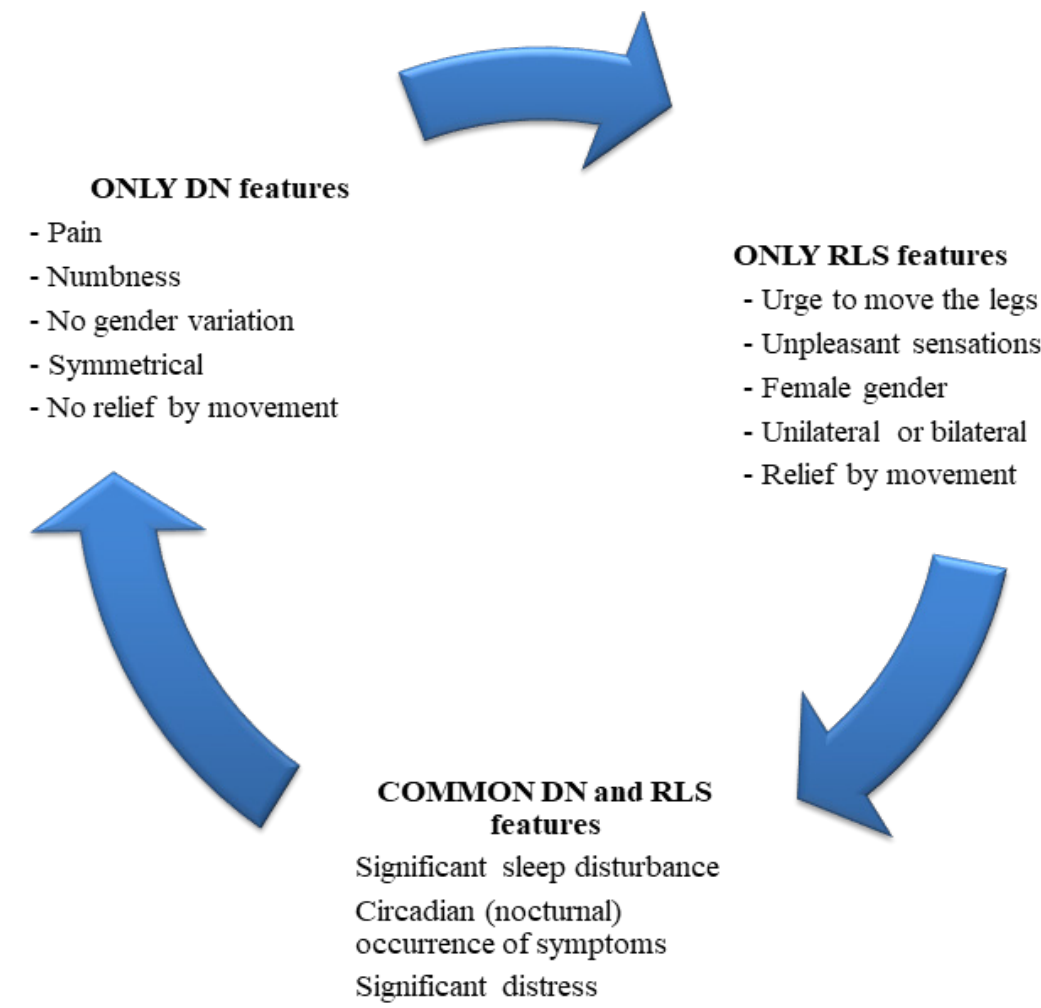

Figure 1. Clinical features 'easy lock' technique to enhance doctors' readiness when DN and RLS co-exist (based on ref 10).

Ruling out other common causes of peripheral neuropathy including alcohol consumption, chemotherapy, B12 deficiency, hypothyroidism, vasculitis, renal diseases, human immune deficiency virus, is necessary $[8,10]$.

The treatment of DN includes both achieving optimal glycemic control and administering medication for pain relief. The most commonly used and most effective studied drugs are: pregabalin, duloxetine and tapentadol [2]. Some patients respond to tricyclic antidepressants, gabapentin, venlafaxine, tramadol, capsaicin [2].

Regarding RLS, treatment contains both nonpharmacological and pharmacological options. Nonpharmacological therapy includes caffeine and tobacco restriction, discontinuation of SSRIs and dopamine antagonists, massage and relaxation techniques [10]. The gold standard of pharmacological therapy is dopamine agonists pramipexole $(0.125-2 \mathrm{mg}$ day) or ropinirole $(0.125-4 \mathrm{mg} /$ day) administrated $1-2$ hours before bedtime [3]. Other medications include bromocriptine, benzodiazepines, codeine, alpha-2-agonists (clonidine) [3].

In this patient with DM, RLS was not recognized and symptoms were falsely attributed to hypoglycemia episodes and peripheral DN, a frequent complication of DM. Symptoms of RLS resulted in chronic insomnia having a significant negative burden on the patient's quality of life. Differentiating DN and RLS poses a clinical dilemma due to the high prevalence of RLS in diabetics. In this case, a chronic condition such as DM triggered clinical inaction in regards to RLS diagnosis. Diagnosis of RLS can be made straight forward in a primary care setting. For this reason a careful evaluation of patients' complains of sleep disturbance, of anxiety, depression, frequent night awakening are of paramount importance in order to formulate RLS diagnosis. Physicians have to be aware of the association between RLS and DM and include the syndrome in their differential diagnosis.

Coexistence of DN and RLS appear uncommon as a diagnostic eventuality, but perhaps clinical 'dynamics' and diagnostic likelihood differ if a female patient with DM occurrence for thirty years, persistent sleep satisfaction loss for almost ten years and low ferritin levels seeks medical advice. Using RLS criteria, a primary care physician can exclude RLS without any need of sophisticated clinical approach if a familiarity on the doctors' part to recall such diagnostic option exists. This becomes more interesting thinking that RLS treatment is available and effective. It is unknown to what 
extent a better glycemic control may occur (directly or indirectly) once sleep is restored. It is also questionable to what extent a known chronic condition can 'bury' other occurring morbidity manifestations.

\section{References}

1. Ohayon MM, O’Hara R, Vitiello MV. Epidemiology of restless legs syndrome: a synthesis of the literature. Sleep Med Rev. 2012;16:283-295.

2. Cuellar NG, Dorn JM. Peripheral diabetic neuropathy or restless legs syndrome in persons with type 2 diabetes mellitus: Differentiating diagnosis in practice. J Am Assoc Nurse Pract. 2015;27:671-675.

3. Symvoulakis E, Anyfantakis D, Lionis C. Restless legs syndrome: literature review. Sao Paulo Med J. 2010;128:167170.

4. Willis T. The London practice of physick. London, England: Basset \& Crooke; 1685.

5. Ekbom KA. Restless legs. Acta Med Scand. 1945;158
Suppl:1-123.

6. Allen RP, Earley CJ. Restless legs syndrome: a review of clinical and pathophysiologic features. J Clin Neurophysiol. 2001;18:128-147.

7. Earley CJ. Clinical practice. Restless legs syndrome. N Engl J Med. 2003;348:2103-2109.

8. Allen RP, Picchietti DL, Garcia-Borreguero D, Ondo WG, Walters AS, Winkelman JW, et al. Restless legs syndrome/Willis-Ekbom disease diagnostic criteria: updated International Restless Legs Syndrome Study Group (IRLSSG) consensus criteria--history, rationale, description, and significance. Sleep Med. 2014;15:860-873.

9. Akın S, Bölük C, Türk Börü Ü, Taşdemir M, Gezer T, Şahbaz FG, et al. Restless legs syndrome in type 2 diabetes mellitus. Prim Care Diabetes. 2019;13:87-91.

10. Kalra S, Gupta A. Diabetic Painful Neuropathy and Restless Legs Syndrome in Diabetes. Diabetes Ther. 2018;9:441-447.

11. Vinik AI, Kong X, Megerian JT, Gozani SN. Diabetic nerve conduction abnormalities in the primary care setting. Diabetes Technol Ther. 2006;8:654-662. 